Meta

Journal des traducteurs

Translators' Journal

\title{
Le piano
}

\section{Alain Morissette}

Volume 21, numéro 2, juin 1976

URI : https://id.erudit.org/iderudit/003667ar

DOI : https://doi.org/10.7202/003667ar

Aller au sommaire du numéro

Éditeur(s)

Les Presses de l'Université de Montréal

ISSN

0026-0452 (imprimé)

1492-1421 (numérique)

Découvrir la revue

Citer cet article

Morissette, A. (1976). Le piano. Meta, 21(2), 143-146.

https://doi.org/10.7202/003667ar

Ce document est protégé par la loi sur le droit d'auteur. L'utilisation des services d'Érudit (y compris la reproduction) est assujettie à sa politique d'utilisation que vous pouvez consulter en ligne.

https://apropos.erudit.org/fr/usagers/politique-dutilisation/
Cet article est diffusé et préservé par Érudit.

Érudit est un consortium interuniversitaire sans but lucratif composé de l’Université de Montréal, l'Université Laval et l'Université du Québec à Montréal. Il a pour mission la promotion et la valorisation de la recherche. https://www.erudit.org/fr/ 


\section{LE PIANO}

A. Aperçu historique. - Selon toute vraisemblance, l'origine du piano moderne remonterait au début des années 1700 .

Avant cette époque, les instruments à clavier et à cordes frappées sont nombreux, mais ils diffèrent sensiblement du piano par le mode d'attaque de la corde. Le psaltérion (psaltery) est, chronologiquement, le premier instrument à cordes pincées avec les doigts, puis, plus tard, frappées à l'aide de petits maillets. A partir du $\mathrm{Xv}^{\mathrm{e}}$ siècle, une panoplie d'instruments procédant du psaltérion se répandent en Europe; leur perfectionnement donne naissance au clavicorde (clavichord) muni d'un clavier dont l'élément percutant est une petite pièce métallique appelée tangente (tangent), qui vient frapper la corde et reste en contact avec elle jusqu'à ce qu'on relève la touche. C'est au début du $\mathrm{XvIII}^{\mathrm{e}}$ siècle que le piano supplante le clavicorde grâce à l'invention de Bartolommeo Cristofori, la mécanique à échappement simple (single action). Quelques années plus tard, le français Sébastien Érard réussit à mettre au point la mécanique à double échappement (double action, double escapement repetition action, double escapement action) sur le principe duquel sont construits les pianos modernes. Au début du XIX ${ }^{e}$ siècle, l'étendue (compass, range) du piano atteint 7 octaves. Les autres innovations des facteurs de piano (piano makers) ne représentent qu'un apport minime, car elles ne modifient pas de façon sensible l'instrument tel que nous le connaissons aujourd'hui.

B. Le piano. - Le mot piano est une forme abrégée de l'italien pianoforte ou fortepiano, instrument à clavier et à cordes frappées (keyboard striking action instrument), capable de produire toutes les nuances, du piano (doux) ou forte (fort).

Le piano se compose essentiellement des parties suivantes : la caisse, le clavier, la mécanique, les marteaux, les cordes, la table d'harmonie, le barrage, le sommier, le châssis et les pédales.

1) La caisse (case) donne, en fait, la forme au piano. Elle a suivi une lente évolution au cours des siècles — piano pyramidal (pyramid piano), piano-lyre (lyre piano), piano-girafe (giraffe piano) qui ne sont plus que des pièces de musée - pour en arriver au piano à queue (grand piano) et au piano droit (upright piano) d'aujourd'hui. La caisse est faite des bois les plus variés: acajou, noyer, ébène, par exemple.

2) Le clavier (keyboard), qui est en quelque sorte l'élément moteur du piano, est constitué par l'ensemble des touches (keys) au nombre de 88. La touche est un petit levier qui, en basculant (being pivoted) sur une pointe de métal, dite pointe de balancier ou point de bascule (pivot point), provoque le heurt du marteau sur la corde.

3) La mécanique (action) est l'ensemble des leviers qui relient la touche au marteau. L'action de la touche sur le marteau et du marteau sur la corde s'effectue au moyen d'un mécanisme appelé échappement (escapement, hopper), par l'intermédiaire du pilote (pilot) articulé dans la touche et dans le grand levier (carriage, 
carriage lever). Ce dernier porte à son extrémité antérieure l'échappement en forme d'équerre (L-shaped hopper). Lorsque le mécanisme est au repos, la branche de l'échappement qui traverse le petit levier (repetition lever) vient porter sous le rouleau (roller) situé sous le manche du marteau (hammer shank). Sur la garniture de repos du marteau (hammer rest) est fixée l'attrape (check, backcheck) qui traverse le manche du marteau près de la tête de celui-ci. L'attrape a pour fonction d'empêcher le flottement du marteau au moment où il vient d'attaquer (strike) la corde.

Étudions maintenant ce qui se passe lorsque la touche a été frappée (depressed). Le pilote soulève simultanément le grand levier et l'échappement qui pousse le marteau vers la corde. La tête du marteau parvenant à proximité de la corde, la branche de l'échappement rencontre le bouton de réglage (adjustable set-off button, set-off button), et l'équerre bascule, abandonnant le marteau qui poursuit seul sa course sous l'impulsion acquise. Au même moment, une petite pièce métallique vient s'appuyer sur le petit levier. Cette disposition assure le bon fonctionnement des notes répétées. Cependant, avec une telle mécanique, le piano ne produit encore qu'un ensemble de sons confus. Afin de se détacher nettement l'une de l'autre ou, au contraire, de se mêler harmonieusement, les notes doivent être étouffées à volonté. À cette fin, chaque note est munie d'une pièce de bois garnie de feutre, l'étouffoir (damper), qui, en position de repos, est appuyée sur la corde pour l'empêcher de vibrer. Le rôle de l'étouffoir consiste en quelque sorte à éteindre la note (silence the string).

4) Les marteaux (hammers), dont la tête s'allonge légèrement en forme de fuseau, sont des pièces de bois recouvertes de plusieurs épaisseurs de feutre pour pouvoir résister au tranchant des cordes.

5) Les cordes (stringing). La longueur, la tension et la texture des cordes sont variables suivant le degré de l'échelle sonore et respectent certaines lois fondamentales de l'acoustique. C'est ainsi que les cordes graves sont plus longues que les cordes aiguës, ce qui explique la forme des pianos à queue. Les cordes du piano sont triples (trichord stringing) dans le registre aigu (treble register) et dans le registre médium (middle register). Les cordes des notes basses sont doubles (bichord stringing) et filées (overspun, covered), c'est-à-dire qu'elles sont recouvertes d'un fil de cuivre, tandis que les extrêmes basses ne comptent qu'une seule corde. À la partie supérieure, les cordes sont rattachées à un cadre métallique. À l'autre extrémité, chaque corde s'enroule autour d'une cheville qui assure le réglage de la tension. Enfin, la méthode des cordes croisées (cross-stringing, overstringing) permet de renforcer les cordes dans un châssis plus petit.

6) La table de résonance ou table d'harmonie (resonating soundboard, soundboard). C'est une surface plane en bois, au-dessus de laquelle sont tendues les cordes et qui a pour but d'en accroître, par sa propre vibration, la sonorité.

7) Le barrage (bracing) est constitué par un ensemble de barreaux réunis par des traverses et encadrés par un châssis. Il supporte la table de résonance et le sommier. 
8) Le sommier (pin block, wrest plank, wrest block). Les cordes sont attachées à l'une des extrémités par des pointes fixées dans le cadre de métal et à l'autre extrémité par des chevilles (wrest pins) enfoncées dans un sommier en bois.

9) Le châssis (frame). C'est un cadre de fer ou de fonte très robuste, qui supporte la tension des cordes.

10) Les pédales (pedals). Tous les pianos sont munis de deux pédales et un bon nombre en comptent trois. Elles permettent de produire les nuances les plus variées. La pédale forte (loud pedal, damper pedal), appelée improprement pédale forte, est placée à droite. Elle permet de soulever uniformément tous les étouffoirs de façon à prolonger la résonance et la durée des sons. La sourdine (soft pedal, una corda pedal) ou pédale douce (calque de l'anglais) est située à gauche. Elle amortit le son par le déplacement du jeu complet des marteaux. Enfin, la pédale de prolongation (sostenuto pedal, centre pedal) au centre, permet de prolonger la résonance des sons du registre grave uniquement.

\section{LEXIQUE}

action
adjustable set-off button
backcheck
bichord stringing
bracing
carriage
carriage lever
case
centre pedal
check
clavichord
compass
covered (string)
cross-stringing
damper
damper pedal
depress (to)
double action
double-escapement action
double escapement repetition action
escapement
frame
giraffe piano
grand piano
hammer
hammer rest
hammer shank
hopper
key
keyboard
keyboard striking action instrument

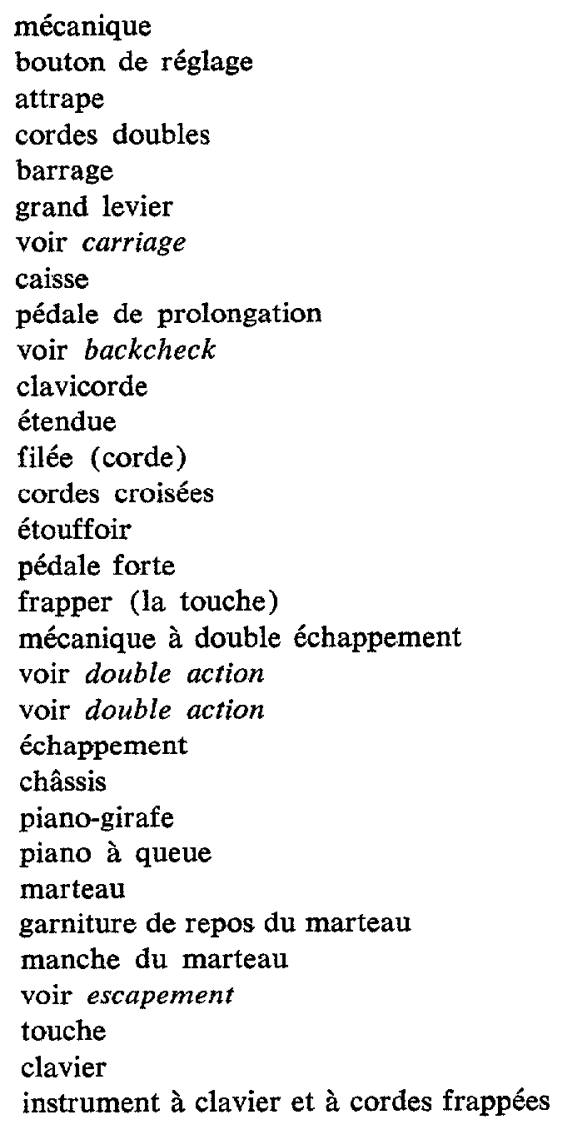




\author{
loud pedal \\ L-shaped hopper \\ lyre piano \\ middle register \\ overspun \\ overstringing \\ piano maker \\ pilot \\ pin block \\ pivoted, be \\ pivot point \\ psaltery \\ pyramid piano \\ range \\ repetition lever \\ resonating soundboard \\ roller \\ set-off button \\ silence the string (to) \\ single action \\ soft pedal \\ sostenuto pedal \\ soundboard \\ strike (to) \\ tangent \\ treble register \\ trichord stringing \\ una corda pedal \\ upright piano \\ wrest block \\ wrest pin \\ wrest plank
}

\author{
voir damper pedal \\ échappement en forme d'équerre \\ piano-lyre \\ registre médium \\ voir covered \\ voir cross-stringing \\ facteur de piano \\ pilote \\ sommier \\ basculer \\ point de bascule, pointe de balancier \\ psaltérion \\ piano pyramidal \\ voir compass \\ petit levier \\ table de résonance, table d'harmonie \\ rouleau \\ voir adjustable set-off button \\ éteindre la note \\ mécanique à échappement simple \\ sourdine \\ voir centre pedal \\ voir resonating soundboard \\ attaquer (la corde) \\ tangente \\ registre aigu \\ cordes triples \\ voir soft pedal \\ piano droit \\ voir pin block \\ cheville \\ voir pin block
}

Alain Morissette

\section{BIBLIOGRAPHIE}

APEL, Willie, Harvard Dictionary of Music, Cambridge (Mass.), Belknap Press of Harvard University Press, 1969, $2^{e}$ ed., 1969, 934 p.

DE CANDÉ, Roland, Dictionnaire de musique, Paris, Seuil, 1970, 283 p., «Dictionnaires microcosmes $\gg, n^{\circ} 1$.

Encyclopaedia Britannica, vol. 17, 1973.

Encyclopaedia Universalis, vol. 13, 1968.

JACOBS, Arthur, A New Dictionary of Music, Harmondsworth (England), Penguin Reference Books, 1972, 425 p.

LOCARD, Paul et Rémy STRICKER, le Piano, Paris, P.U.F., 1966, 120 p. «Que sais-je ?», $n^{\circ} 263$.

MICHEL, François, Encyclopédie de la musique, t. III, Paris, Fasquelle, 1961, 1023 p. 